\title{
miR-30d inhibits cell biological progression of Ewing's sarcoma by suppressing the MEK/ERK and PI3K/Akt pathways in vitro
}

\author{
CONGLIN YE, XIAOLONG YU, XUQIANG LIU, MIN DAI and BIN ZHANG \\ Department of Orthopedics, The First Affiliated Hospital of Nanchang University, Artificial Joints Engineering \\ and Technology Research Center of Jiangxi Province, Nanchang, Jiangxi 330006, P.R. China
}

Received June 8, 2017; Accepted January 3, 2018

DOI: $10.3892 / \mathrm{ol} .2018 .7900$

\begin{abstract}
MicroRNAs (miRNAs) are small, single-stranded, non-coding RNA molecules involved in cancer initiation and progression. The present study aimed to determine the effect of miRNA-30d (miR-30d) on the growth, malignant phenotype, and apoptosis of Ewing's sarcoma (ES) SK-ES-1 cells, and to elucidate the underlying molecular mechanism and signaling pathway involved. Cell proliferation, invasion, migration, morphological changes, cell cycle distribution and apoptosis were investigated. Furthermore, the expression of matrix metalloproteinase (MMP)-2, MMP-9, B-cell lymphoma 2 (Bcl-2), Bcl-2-associated $\mathrm{X}$ protein (Bax), caspase-3 and poly (ADP-ribose) polymerase (PARP) were examined, as was the activity of the mitogen-activated protein kinase kinase (MEK)/extracellular signal-regulated kinase (ERK) and phosphoinositide 3-kinase (PI3K)/Akt pathways. It was found that the overexpression of miR-30d repressed the proliferation, migration and invasion, and promoted morphological changes, S-phase arrest and apoptosis of SK-ES-1 cells. Additionally, it was observed that increased miR-30d levels inhibited the expression of MMP-2 and MMP-9, and inhibited the activity of the MEK/ERK and PI3K/Akt pathways, but elevated the ratio of Bax/Bcl-2 and the cleavage of caspase-3 and PARP. Taken together, the results demonstrated that miR-30d suppressed the biological progression of SK-ES-1 cells by targeting MMP-2 and MMP9, the Bax/Bcl-2 and caspase- 3 cascade, and the MEK/ERK and PI3K/Akt signaling pathways. Therefore, miR-30d is a promising target in the treatment of ES. However,
\end{abstract}

Correspondence to: Dr Bin Zhang or Professor Min Dai, Department of Orthopedics, The First Affiliated Hospital of Nanchang University, Artificial Joints Engineering and Technology Research Center of Jiangxi Province, 17 Yong Wai Zheng Street, Nanchang, Jiangxi 330006, P.R. China

E-mail: 1294522934@qq.com

E-mail: daimin@medmail.com.cn

Key words: microRNA-30d, Ewing's sarcoma, invasion and migration, apoptosis, mitogen-activated protein kinase kinase/extracellular signal-regulated kinase, phosphoinositide 3-kinase/Akt further investigations are urgently required to investigate the underlying molecular mechanisms of the effects of miR-30d on ES for a comprehensive understanding of the tumorigenesis and progression of this cancer.

\section{Introduction}

Ewing's sarcoma (ES), the second most frequent bone tumor, mainly affects children and adolescents (1). ES is an aggressive and metastatic tumor type; approximately one-third of patients with ES present with metastasis at diagnosis, resulting in poor prognosis. The 5-year survival rate of patients with metastatic ES ranges between 20 and $45 \%$, depending on the metastatic location, compared with $60-70 \%$ in those with localized disease (2). Therefore, an improved understanding of the biology of this malignancy is crucial to the development of innovative therapeutic strategies, the identification of novel therapeutic targets, and ultimately the improvement of patient prognosis.

MicroRNAs (miRNAs or miRs) are a specific type of small, non-coding RNA, which regulate gene expression by suppressing mRNA translation. The aberrant expression of miRNAs contributes to tumor initiation and progression, and affects prognosis (3-5). Previously, numerous studies have indicated that miRNAs are important in the initiation and progression of ES (6-8). Of these cancer-associated miRNAs, miR-30d has been shown to exert antitumor effects in a several types of human cancer (9-11). However, it has been reported that high tissue expression of miR-30d predicts poor prognosis in patients with prostate cancer (12). To date, the exact effects of miR-30d on the progression of ES, and the underlying mechanisms, remain to be fully elucidated.

In the present study, ES SK-ES-1 cells were transfected with an miR-30d mimic to examine the effects of miR-30d on cell proliferation, invasion, migration and apoptosis, and to elucidate the underlying molecular mechanisms. To the best of our knowledge, the present study represents the first attempt to demonstrate the effects of miR-30d on the biological progression of ES in vitro.

\section{Materials and methods}

Materials and reagents. The SK-ES-1 human ES cell line was obtained from the American Type Culture Collection (Manassas, VA, USA). Scrambled-sequence mimic and 
miR-30d mimic were purchased from Biotend (Shanghai, China). Lipofectamine 2000 and OPTI-MEM were purchased from Invitrogen; Thermo Fisher Scientific, Inc. (Waltham, MA, USA). RPMI 1640 medium, fetal bovine serum (FBS), phosphate-buffered saline (PBS), dimethyl sulfoxide (DMSO), bovine serum albumin (BSA) and Cell Counting Kit- 8 (CCK-8) were purchased from Beijing Transgen Biotech Co., Ltd. (Beijing, China). The Cycletest Plus DNA Reagent kit, Annexin-V/FITC kit and Matrigel were purchased from BD Biosciences (San Jose, CA, USA). The Transwell invasion chambers were purchased from Costar (Cambridge, MA, USA). Crystal violet staining solution was purchased from Beyotime Institute of Biotechnology (Haimen, China). A Hoechst 33258 staining kit was purchased from Nanjing Keygen Biotech Co., Ltd. (Nanjing, China). Antibodies against MMP-2 (cat. no. 40994), MMP-9 (cat. no. 13667), Bax (cat. no. 5023), Bcl-2 (cat. no. 4223), caspase-3 (cat. no. 9665), PARP (cat. no. 9532), MEK1/2 (cat. no. 8727), ERK1/2 (cat. no. 4695), phosphorylated MEK1/2 (p-MEK1/2) (cat. no. 9154), phosphorylated ERK1/2 (p-ERK1/2) (cat. no. 4370), phosphoinositide 3-kinase (PI3K) (cat. no. 4249), Akt (cat. no. 4691), phosphorylated Akt (p-Akt) (cat. no. 4060) and $\beta$-actin (cat. no. 4970) were purchased from Cell Signaling Technology, Inc. (Danvers, MA, USA). Horseradish peroxidase-conjugated secondary antibodies (cat. no. HS101-01) were purchased from Beijing Transgen Biotech Co., Ltd. (Beijing, China).

Cell culture and transfection. The SK-ES-1 cells were grown in RPMI 1640 medium supplemented with 10\% (v/v) FBS, $100 \mathrm{U} / \mathrm{ml}$ penicillin, and $100 \mu \mathrm{g} / \mathrm{ml}$ streptomycin. The cells were maintained at $37^{\circ} \mathrm{C}$ in an incubator containing $5 \% \mathrm{CO}_{2}$ and passaged every 2-3 days. All cells used in the present study were subjected to $<15$ passages. Cells at logarithmic phase were seeded in 6-well plates at a density of $3 \times 10^{5}$ cells/well and cultured overnight prior to transfection. Subsequently, transfections were performed for the scrambled-sequence mimic and miR-30d mimic (Biotend) using Lipofectamine 2000 reagent, according to the manufacturer's protocol. The culture medium was changed from OPTI-MEM to RPMI 1640 medium containing $10 \%$ FBS $5 \mathrm{~h}$ following transfection. After 24 or $48 \mathrm{~h}$, the transfected cells were collected and used in subsequent experiments.

Cell proliferation assay. For the analysis of cell proliferation, the cells were seeded into 96 -well plates at $3 \times 10^{3}$ cells/well, and incubated in $10 \%$ CCK- 8 diluted in normal culture medium at $37^{\circ} \mathrm{C}$ until visual color conversion was observed. Proliferation was examined at 24,48 and $72 \mathrm{~h}$ post-transfection by measuring the absorbance in each well at $450 \mathrm{~nm}$ using a universal microplate reader (EL800; BioTek Instruments, Inc., Winooski, VT, USA). All experiments were repeated three times.

Boyden chamber Transwell assays. The invasion capacity of the SK-ES-1 cells was determined using Matrigel-coated Transwell cell culture chambers (pore size, $8 \mu \mathrm{m}$ ). At $24 \mathrm{~h}$ post-transfection, $5 \times 10^{4}$ cells suspended in serum-free RPMI 1640 medium were added to the upper chamber, and medium containing $20 \%$ FBS was added to the lower chamber as chemoattractant. After $24 \mathrm{~h}$, non-invading cells on the upper membrane surface were removed using a cotton swab, whereas invading cells on the lower membrane surface were fixed, stained with crystal violet staining solution, and counted under a phase-contrast microscope (Olympus Corporation, Tokyo, Japan) in three randomly selected fields (magnification, x100). The experiments were performed in triplicate.

Wound healing assays. The migration of SK-ES-1 cells was measured using wound healing assays. The cells were grown in 6-well plates and transfected with the scrambled-sequence mimic and miR-30d mimic. At 24 h post-transfection, linear scratch wounds were created on the confluent cell monolayers using $100 \mu \mathrm{l}$ pipette tips. To prevent cells from entering the cell cycle prior to wounding, they were cultured in serum-free medium. Scratch wounds in the three groups were observed using a phase-contrast microscope and images were captured at 0 and $24 \mathrm{~h}$ (magnification, $\mathrm{x} 40$ ). Experiments were repeated independently three times.

Hoechst 33258 staining of SK-ES-1 cells. At $48 \mathrm{~h}$ post-transfection, cells of the three groups in 6-well plates were fixed with $4 \%$ paraformaldehyde for $30 \mathrm{~min}$ at $25^{\circ} \mathrm{C}$, washed three times with ice-cold PBS, and stained with $10 \mathrm{mg} / \mathrm{l}$ Hoechst 33258 for $10 \mathrm{~min}$ in the dark at $25^{\circ} \mathrm{C}$. Subsequently, the stained nuclei were observed under a fluorescence microscope (Olympus Corporation) with excitation at $350 \mathrm{~nm}$ and emission at $460 \mathrm{~nm}$ (magnification, x200). The experiments were performed in triplicate.

Cell cycle analysis. Cell cycle was analyzed via flow cytometry $48 \mathrm{~h}$ post-transfection. The transfected cells of the three groups were collected and stained with propidium iodide (PI) using a Cycletest Plus DNA Reagent kit, according the manufacturer's protocol. Cell cycle distribution was analyzed using a FACSVerse flow cytometer (BD Biosciences). The percentages of cells in each phase of the cell cycle were compared. The experiments were performed in triplicate.

Cell apoptosis analysis. The cell apoptosis assays were performed using an Annexin-V/FITC kit. Cell apoptosis was detected by flow cytometry $48 \mathrm{~h}$ post-transfection. The transfected cells were collected and washed in ice-cold PBS prior to being stained with Annexin-V/FITC and PI solution for $15 \mathrm{~min}$ in the dark. The ratios of apoptotic cells were determined using a FACSVerse flow cytometer. All experiments were repeated three times.

Western blot analysis. Following transfection, the SK-ES-1 cells were grown in 6-well plates and incubated in RPMI 1640 medium with $10 \%$ FBS for $48 \mathrm{~h}$. Subsequently, the cells of the three groups were collected and lysed in RIPA buffer containing PMSF and phosphatase inhibitor cocktail. Each sample was centrifuged at $17,105.6 \mathrm{~g}$ for $10 \mathrm{~min}$ at $4^{\circ} \mathrm{C}$ to remove cell debris using UNIVERSAL 320R (Hettich Corp., Tuttlingen, Germany), and the supernatant was collected for immunoblotting. The protein concentrations were calculated using BSA as the standard. Proteins $(10 \mu \mathrm{l})$ were loaded and separated by electrophoresis on $10 \%$ SDS-polyacrylamide gels at $110 \mathrm{~V}$ for $2 \mathrm{~h}$ under reducing conditions. Following electrophoresis, the proteins were transferred onto PVDF membranes in a Tris-glycine transfer buffer, and incubated with antibodies 
A
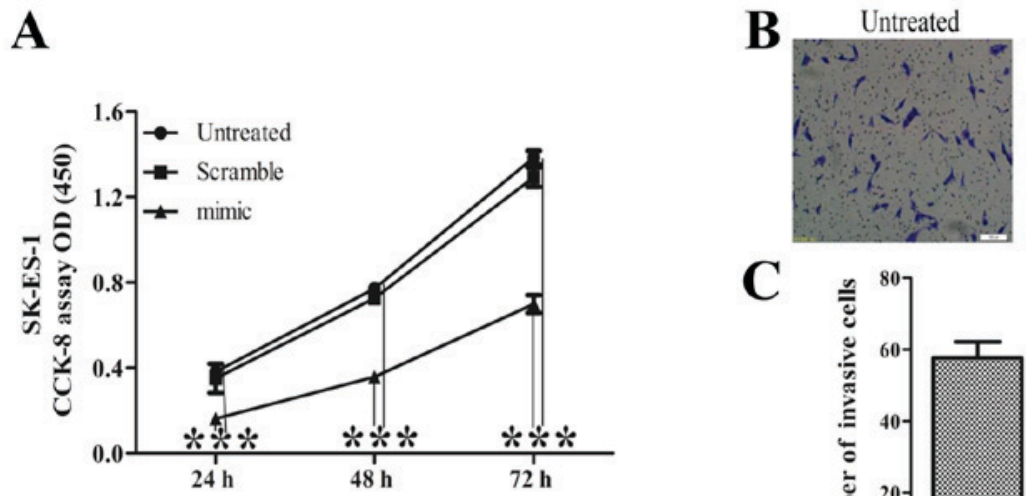

C
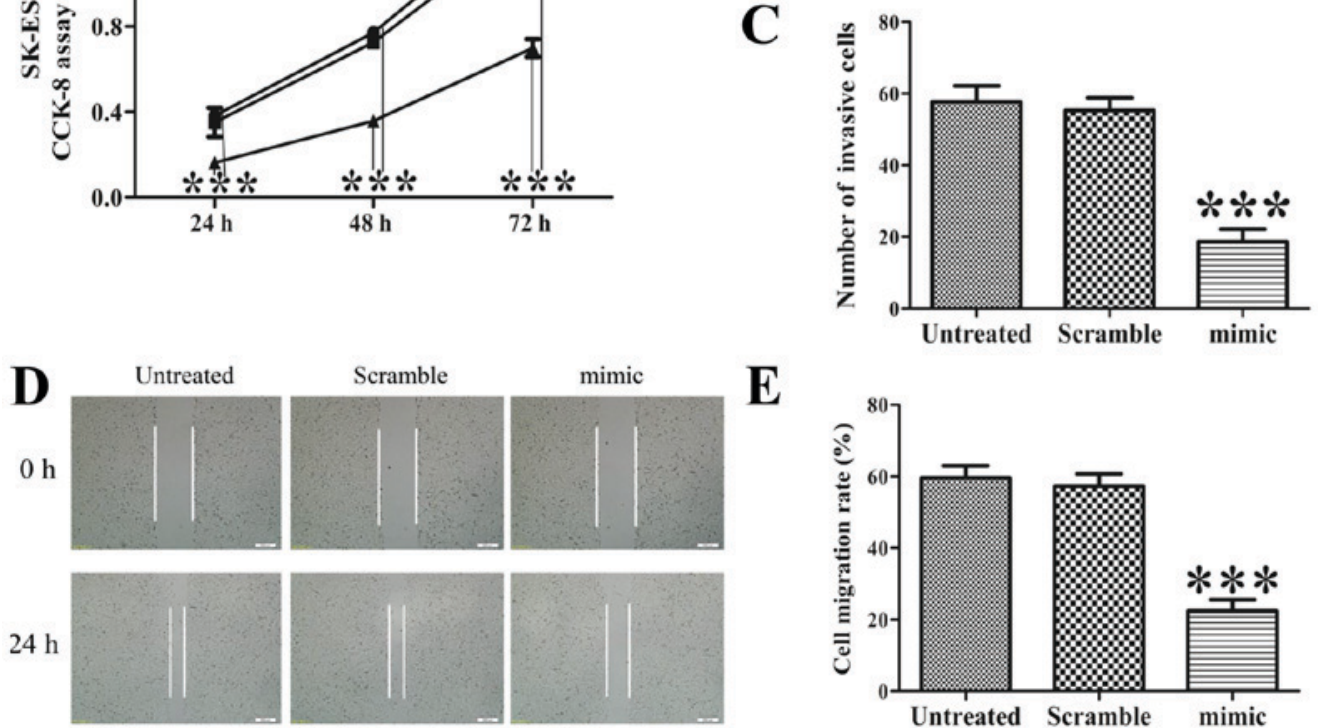

$\mathbf{E}$

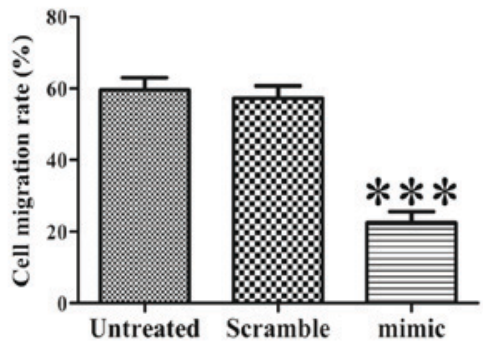

Figure 1. Increased miR-30d inhibits proliferation, migration and invasion of SK-ES-1 cells. (A) CCK-8 assay revealed that ectopic miR-30d significantly suppressed cell growth in SK-ES-1 cells $\left({ }^{* * *} \mathrm{P}<0.001\right.$, vs. untreated). Transwell assay (B) images (magnification, $\left.\mathrm{x} 100\right)$ and $(\mathrm{C})$ quantification demonstrated that invasive potential was significantly reduced in the miR-30d mimic group relative to the untreated group ( ${ }^{* * *} \mathrm{P}<0.001$, vs. untreated). Wound healing assay (D) images (magnification, $\mathrm{x} 40$ ) and (E) quantification showed that the overexpression of miR-30d markedly repressed the migration of SK-ES-1 cells compared with the control group $\left({ }^{* * *} \mathrm{P}<0.001\right.$, vs. untreated). miR, microRNA; CCK-8, Cell Counting Kit-8.

against MMP-2 (1:1,000), MMP-9 (1:1,000), Bax $(1: 1,000)$ Bcl-2 (1:1,000), caspase-3 (1:1,000), PARP (1:1,000), MEK1/2 (1:1,000), ERK1/2 (1:1,000), p-MEK1/2 (1:1,000), p-ERK1/2 $(1: 1,000)$, PI3K $(1: 1,000)$, Akt $(1: 1,000)$, p-Akt $(1: 1,000)$ and $\beta$-actin $(1: 1,000)$ overnight at $4^{\circ} \mathrm{C}$. The PVDF membranes were washed in TBST for $10 \mathrm{~min}$ three times, following which secondary HRP-conjugated antibodies $(1: 2,000)$ were added at 1:5,000 dilution and incubated for $2 \mathrm{~h}$ at $25^{\circ} \mathrm{C}$. The PVDF membranes were subsequently washed three times in TBST. Immunoreactive proteins were detected by enhanced chemiluminescence (ECL kit; Beijing Transgen Biotech Co., Ltd.) followed by exposure to X-ray films. The western blot signals were semi-quantified by densitometry using ImageQuant TL software 7.0 (GE Healthcare Life Sciences, Chalfont, UK). All western blot experiments were repeated at least three times.

Statistical analysis. SPSS 19.0 software (IBM SPSS, Armonk, NY, USA) was used for statistical analysis of data. Quantitative data are expressed as the mean \pm standard deviation. Independent two-sample t-tests were used to compare differences between the two groups. One-way analysis of variance with a multiple comparison test was used to analyze differences between three or more groups. $\mathrm{P}<0.05$ was considered to indicate a statistically significant difference.

\section{Results}

Overexpression of miR-30d inhibits the proliferation of SK-ES-1 cells. The effect of the overexpression of miR-30d on
SK-ES-1 cell growth was determined using a CCK-8 assay. As shown in Fig. 1A, the increased expression of miR-30d significantly suppressed cell growth of the SK-ES-1 cells $(\mathrm{P}<0.001)$.

Increased expression of miR-30d represses the invasion and migration of SK-ES-1 cells. Boyden chamber Transwell and wound healing assays were performed to determine the effect of ectopic expression of miR-30d on the invasion and migration of SK-ES-1 cells. Representative images of the Transwell filters are shown in Fig. 1B. The invasive cell count, shown in Fig. 1C, demonstrated that the invasive potential was significantly reduced in the miR-30d mimic group, compared with that in the untreated group $\mathrm{P}<0.001)$. In addition, the overexpression of miR-30d led to a decrease in migration capability, as shown in Fig. 1D and $\mathrm{E}(\mathrm{P}<0.001)$.

Western blot assays were performed to examine the effect of increased levels of miR-30d on the expression of MMP-2 and MMP-9, which are closely linked with tumor invasion and metastasis (13-15). As shown in Fig. 2A and B, the expression levels of MMP-2 and MMP-9 were significantly reduced in the miR-30d mimic group compared with those in the untreated group $(\mathrm{P}<0.01)$. These findings suggested that the overexpression of miR-30d inhibits the invasion and migration of SK-ES-1 cells by suppressing the expression of MMP-2 and MMP-9.

Upregulation of miR-30d induces morphological changes in SK-ES-1 cells. Staining with the fluorescent DNA-binding dye Hoechst 33258 revealed that the SK-ES-1 cells transfected 

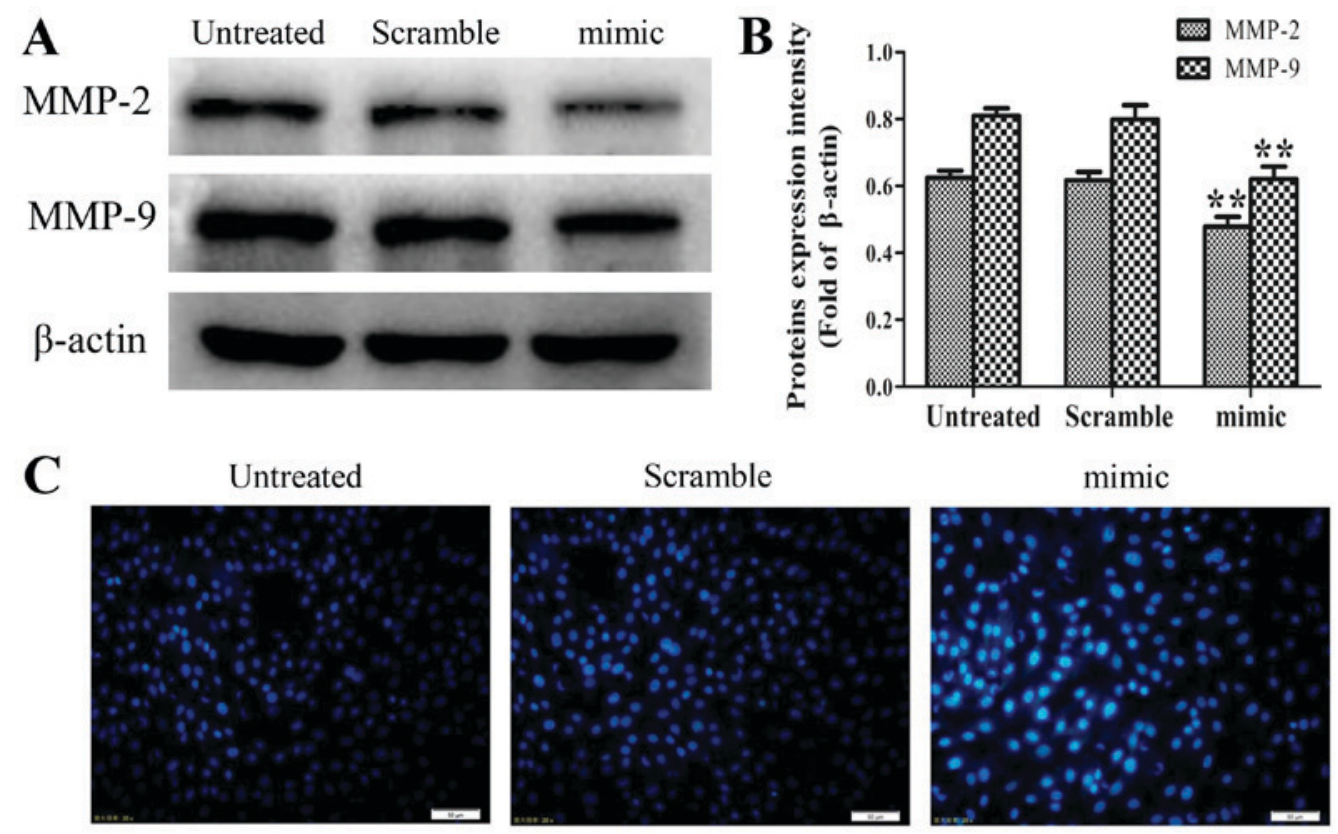

Figure 2. Abnormal miR-30d reduces the expression levels of MMP-2 and MMP-9, and induces morphological changes in SK-ES-1 cells. (A) Blots and (B) quantification of the western blot assay revealed that the expression levels of MMP-2 and MMP-9 were significantly decreased in the miR-30d mimic group compared with those in the untreated group $\left.{ }^{(*} \mathrm{P}<0.01\right)$. (C) Staining with the fluorescent DNA-binding dye Hoechst 33258 revealed that SK-ES-1 cells transfected with the miR30d mimic possessed condensed and fragmented nuclei, which are typical morphological features of apoptotic cells. By contrast, no morphological signs of apoptosis were observed in cells of the untreated group or scrambled-sequence mimic group (magnification, x200). miR, microRNA; MMP, matrix metalloproteinase.
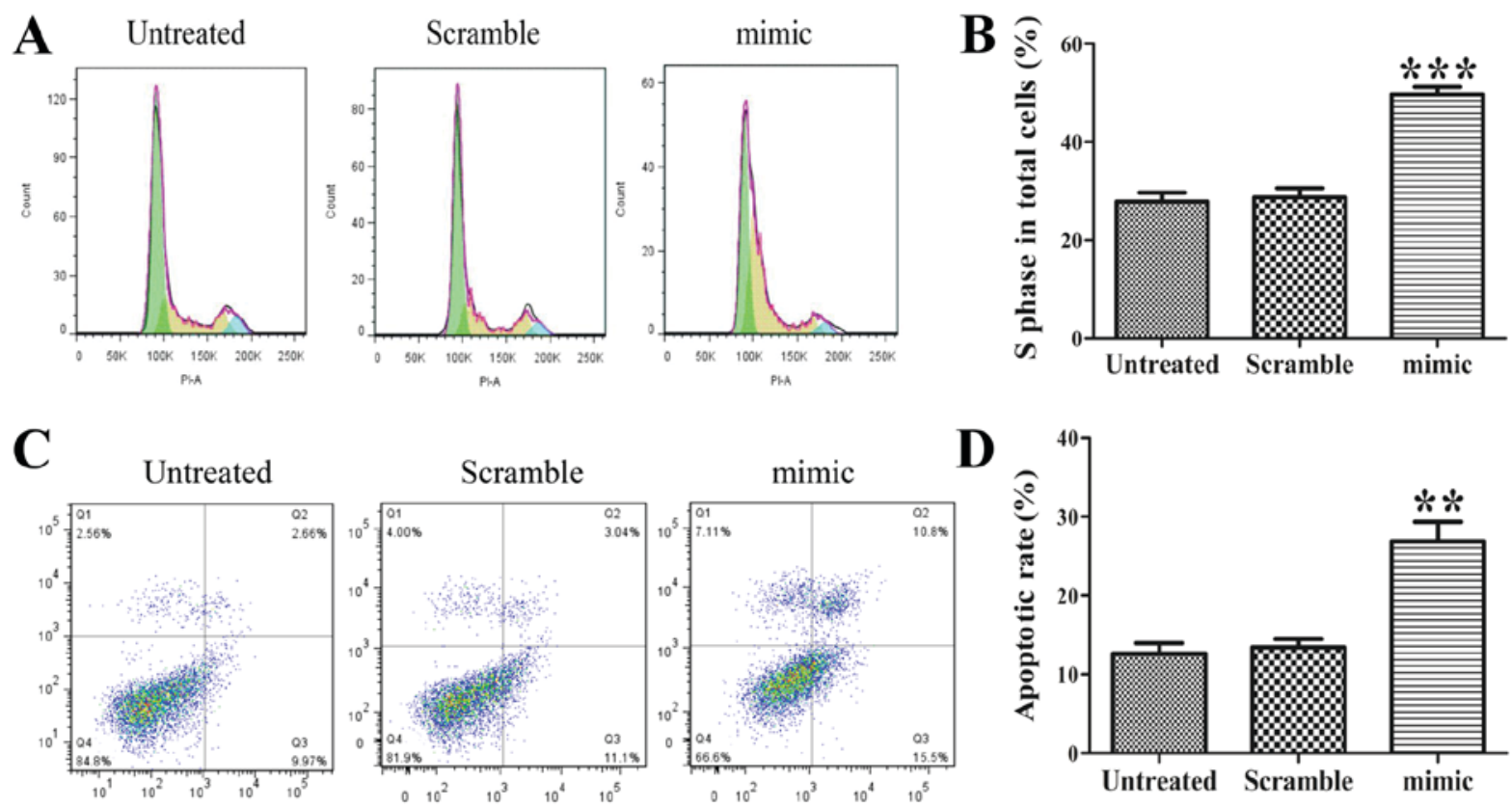

Figure 3. Overexpression of miR-30d arrests the cell cycle at the S phase and leads to apoptosis of SK-ES-1 cells. (A) Flow cytometric assay showed that abnormal miR-30d led to the accumulation of S-phase SK-ES-1 cells and the (B) statistical analysis revealed a significant difference in S-phase cell number between the miR-30d mimic group and the untreated group $\left({ }^{* * *} \mathrm{P}<0.001\right)$. (C) Flow cytometric assay and (D) statistical analysis showed that the proportions of early and late apoptotic cells were significantly elevated in the miR-30d mimic group, compared with those in the untreated group $\left({ }^{* *} \mathrm{P}<0.01\right)$. miR, microRNA.

with the miR30d mimic possessed condensed and fragmented nuclei, which are typical morphological features of apoptotic cells. By contrast, no morphological signs of apoptosis were observed in the cells of the untreated group and scrambled-sequence mimic group. These results indicated that the ectopic expression of miR-30d induced cell death via apoptosis (Fig. 2C).

Ectopic expression of miR-30d arrests the cell cycle at the S-phase. As proliferation is directly linked to cell cycle 
A Untreated Scramble mimic

Bax

Bcl-2
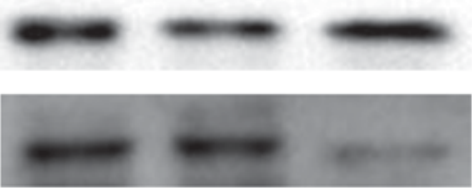

$\beta$-actin

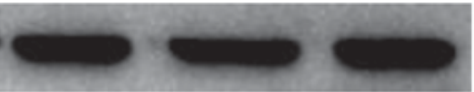

C

Caspase-3

PARP

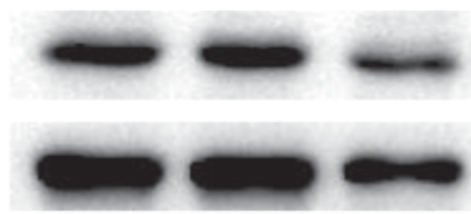

$\beta$-actin

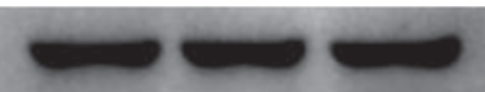

B

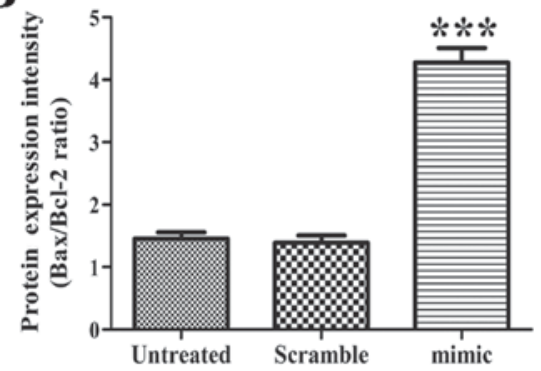

D

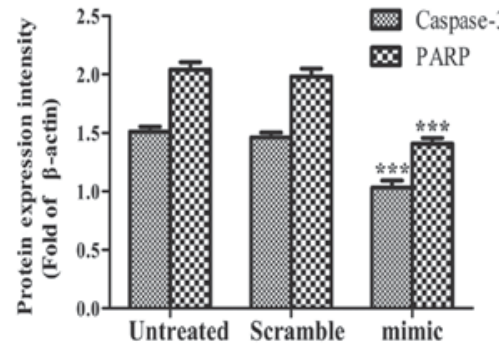

Figure 4. Elevated miR-30d increases the ratio of Bax/Bcl-2 and activates the cleavage of caspase-3 and PARP. (A) Increased miR-30d elevated the expression of Bax and caused a marked reduction in the level of Bcl-2. (B) Bax/Bcl-2 ratio in the miR-30d mimic group was increased (*** $\mathrm{P}<0.001)$. (C) Overexpression of miR-30d led to a marked increase in the cleavage of caspase-3 and PARP with (D) statistical significance, compared with that in the untreated group ( $\left.{ }^{* * *} \mathrm{P}<0.001\right)$. miR, microRNA; Bcl-2, B-cell lymphoma 2; Bax, Bcl-2-associated X protein; PARP, poly (ADP-ribose) polymerase

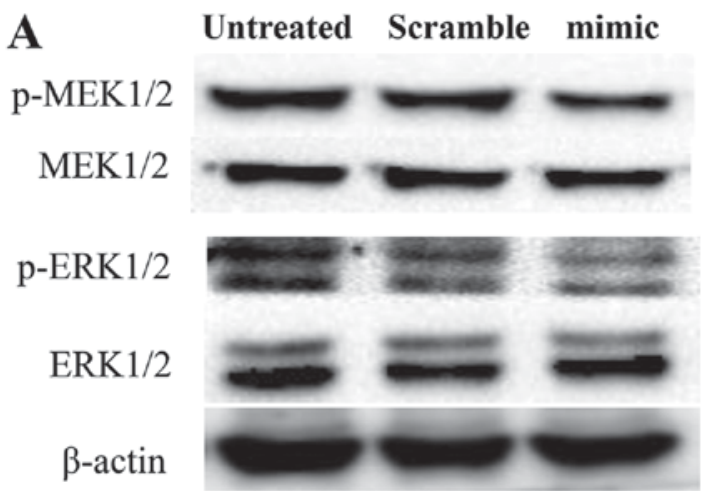

B

$\mathbf{C}$

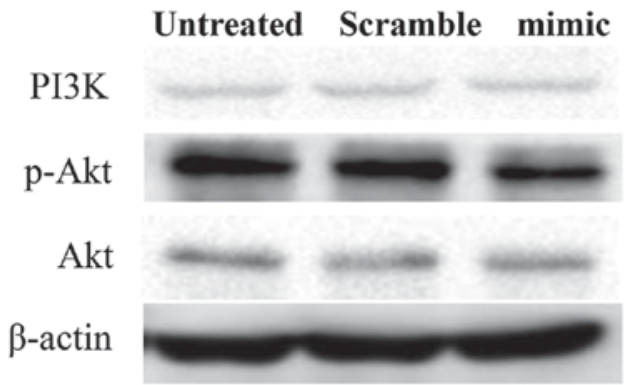

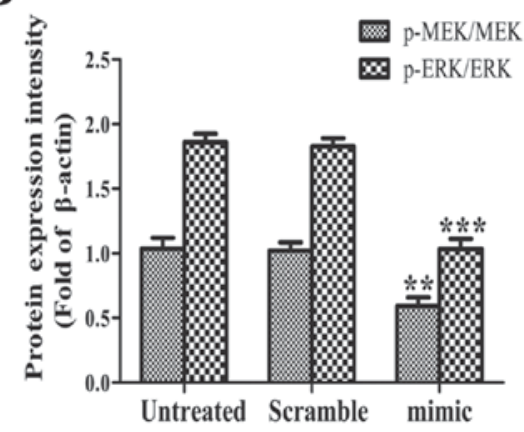

D

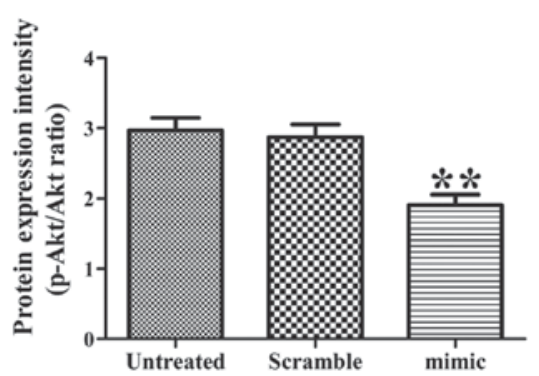

Figure 5. Elevated miR-30d suppresses the MEK/ERK and PI3K/Akt signaling pathways in SK-ES-1 cells. (A) Western blot assay revealed that overexpression of miR-30d reduced the expression levels of p-MEK1/2 and p-ERK1/2, but caused no changes in the levels of MEK1/2 and ERK1/2. (B) Ratios of p-MEK/MEK and p-ERK/ERK in miR-30d mimic group were decreased compared with those in the untreated group $\left({ }^{* *} \mathrm{P}<0.01,{ }^{* * *} \mathrm{P}<0.001\right)$. $(\mathrm{C})$ Expression of p-Akt was decreased in the miR-30d mimic group, but no changes were observed in the levels of PI3K and Akt, compared with those in the untreated group. (D) $\mathrm{p}$-Akt/Akt ratio in the miR-30d mimic group was reduced compared with that in the untreated group ( $\left.{ }^{* *} \mathrm{P}<0.01\right)$. miR, microRNA; MEK, mitogen-activated protein kinase kinase; ERK, extracellular signal-regulated kinase; PI3K, phosphoinositide 3-kinase; p-, phosphorylated.

distribution, the effect of the miR-30d mimic on cell cycle progression was analyzed. The results showed that abnormal miR-30d led to an accumulation of SK-ES-1 cells in the S-phase
(Fig. 3A). The statistical analysis revealed a significant difference in the proportion of S-phase cells between the miR-30d mimic group and the untreated group (Fig. $3 \mathrm{~B}, \mathrm{P}<0.001$ ). 
Overexpression of $\mathrm{miR}-30 \mathrm{~d}$ promotes the apoptosis of $S K-E S-1$ cells. Flow cytometric assays were performed to determine whether the increased expression of miR-30d induced apoptosis of the SK-ES-1 cells. The flow cytometric analysis showed that the proportions of early and late apoptotic cells were significantly elevated in the miR-30d mimic group compared with those in the untreated group (Fig. 3C and D, $\mathrm{P}<0.01)$. Therefore, the ectopic expression of miR-30d induced apoptosis of the SK-ES-1 cells.

Among the 11 known members of the caspase family, which are responsible for key aspects of apoptosis-induced cell death, caspase- 3 is considered to be an executioner in caspase cascades and to have a primary role in apoptosis (16). Following caspase-3 activation, specific substrates for caspase-3, including PARP, are cleaved, which are pivotal for the occurrence of apoptosis $(17,18)$. In addition, changes in the ratio of Bcl-2 family proteins are closely associated with an imbalance in mitochondrial homeostasis, which results in apoptosis $(19,20)$. Elevated levels of pro-apoptotic Bax and/or reduced levels of anti-apoptotic Bcl-2 lead to loss of mitochondrial membrane potential, which is a key process in the initiation of apoptosis (21).

To elucidate the mechanism underlying apoptosis, Bax, Bcl-2, caspase-3 and PARP were detected by western blot analysis. As shown in Fig. 4A, the abnormal expression of miR-30d elevated the expression of Bax, but caused a marked reduction in the level of $\mathrm{Bcl}-2$. The ratio of $\mathrm{Bax} / \mathrm{Bcl}-2$ in the miR-30d mimic group was accordingly increased (Fig. 4B, $\mathrm{P}<0.001)$. The overexpression of miR-30d led to a marked increase in the cleavage of caspase- 3 and PARP compared with that in the untreated group (Fig. $4 \mathrm{C}$ and D, $\mathrm{P}<0.001$ ).

Elevated miR-30d suppresses the MEK/ERK and PI3K/Akt signaling pathways in SK-ES-1 cells. MEK/ERK, a signaling pathway constitutively activated in ES, is involved in several cellular processes, including survival, proliferation, cell cycle regulation, migration, differentiation, metabolism and protein expression $(22,23)$. The PI3K/AKT pathway mediates survival signaling in ES cells $(24,25)$. In the present study, the protein expression levels of p-MEK1/2, MEK1/2, p-ERK1/2, ERK1/2, PI3K, p-Akt and Akt were examined in the three groups by western blot analysis. The results revealed that the overexpression of miR-30d reduced the expression levels of $\mathrm{p}-\mathrm{MEK} 1 / 2$, p-ERK1/2 and p-Akt, but caused no changes in the levels of MEK1/2, ERK1/2, PI3K or Akt. The ratios of p-MEK/MEK, p-ERK/ERK and p-Akt/Akt in the miR-30d mimic group were accordingly decreased, compared with those in the untreated group (Fig. 5A-D, $\mathrm{P}<0.01$ and $\mathrm{P}<0.001$ ). These findings demonstrated that miR-30d acts as an upstream mediator in the MEK/ERK and PI3K/Akt pathways in SK-ES-1 cells.

\section{Discussion}

ES, an aggressive bone and soft tissue malignant tumor principally affecting children and young adults, is characterized by small round cells $(26,27)$. Owing to the development of multi-agent systemic chemotherapy and aggressive local control methods, the overall survival rate of patients with localized disease has risen over the last few decades. Despite these advances, the high rates of relapse and metastasis, and poor prognosis of patients with ES necessitates the development of novel treatments to improve patient outcomes $(26,28)$. A more detailed understanding of the biology of this malignancy is essential for the identification of novel therapeutic targets.

miRNAs are small, non-coding RNA molecules with a length of $\sim 18-25$ nucleotides. miRNAs comprise a large family of non-coding, single-stranded RNAs, which are highly conserved in the majority of eukaryotic organisms $(29,30)$. Through complementary binding to 3'-untranslated regions of target mRNAs, miRNAs regulate gene expression either by suppressing translation or by directly inducing the degradation of the target mRNA (31). Increasing evidence shows that miRNAs are involved in multiple physiological and pathological processes, including human malignancies $(32,33)$. Functionally, miRNAs act as either oncogenes or as tumor suppressors in various types of malignancy (34).

miR-30d, a member of the newly identified miR-30 family, is implicated in tumor development and progression, however, its precise function in this context remains controversial. miR-30d is reported to act as a tumor suppressor in renal carcinoma and anaplastic thyroid carcinoma $(10,35)$, and as an oncogene in melanoma and hepatocellular carcinoma $(36,37)$. To date, there have been no reports of the exact role of miR-30d in the proliferation, malignant phenotype and apoptosis of ES cells, or the underlying molecular mechanisms.

In the present study, it was demonstrated that increased expression of miR-30d inhibited the proliferation, migration and invasion of ES cells, and promoted morphological changes, S-phase arrest and apoptosis in this cell type. Additionally, it was observed that aberrant expression of miR-30d suppressed the expression of MMP-2 and MMP-9, and inhibited the activity of the MEK/ERK and PI3K/Akt pathways. The overexpression of miR-30d also increased the ratio of Bax/Bcl-2 and promoted the cleavage of caspase- 3 and PARP. These findings confirmed that ectopic miR-30d induced cell apoptosis via the Bax/Bcl-2 and caspase- 3 cascade, and that miR-30d is likely to act as an upstream mediator in the MEK/ERK and PI3K/Akt pathways in SK-ES-1 cells.

There were certain limitations to the present study. First, knockdown of the expression of miR-30d was not performed, which may provide confirmation of the effect of miR-30d on SK-ES-1 cells. Second, the present study did not exclude the possibility that the apoptotic effect may result in the suppression of proliferation, invasion and migration. Additionally, the present study was more observational than an in-depth investigation of the mechanism. There was no detailed examination of the molecular mechanism concerning the findings observed. For example, no experiments were performed to determine whether the inhibition of ERK or AKT caused S-phase arrest. In addition, only S473 was examined for AKT phosphorylation, which usually consists of S473 and T307. To confirm the target gene of miR-30d, a dual luciferase reporter assay is also required. Therefore, further investigations are urgently required to examine the underlying molecular mechanisms in detail.

In conclusion, the findings observed in SK-ES-1 cells suggested that miR-30d inhibited the biological progression of ES in vitro by suppressing the MEK/ERK and PI3K/Akt pathways. Therefore, miR-30d is a promising novel therapeutic target for treating ES. However, further investigations are 
urgently required to examine the underlying molecular mechanisms of the effects of miR-30d on ES for a comprehensive understanding of the tumorigenesis and progression of this type of cancer.

\section{Acknowledgements}

The present study was supported by the Foundation of the Health Department of Jiangxi Province on Traditional Chinese Medicine (grant no. 2016A073) and Gan-Po Talents Project 555 of Jiangxi Province.

\section{Competing interests}

The authors declare that they have no competing interests.

\section{References}

1. Jemal A, Bray F, Center MM, Ferlay J, Ward E and Forman D: Global cancer statistics. CA Cancer J Clin 61: 69-90, 2011.

2. Gaspar N, Hawkins DS, Dirksen U,Lewis IJ, Ferrari S, Le Deley MC, Kovar H, Grimer R, Whelan J, Claude L, et al: Ewing sarcoma: Current management and future approaches through collaboration. J Clin Oncol 33: 3036-3046, 2015.

3. Medina-Villaamil V, Martínez-Breijo S, Portela-Pereira P, Quindós-Varela M, Santamarina-Caínzos I, Antón-Aparicio LM and Gómez-Veiga F: Circulating microR NAs in blood of patients with prostate cancer. Actas Urol Esp 38: 633-639, 2014.

4. Yang M, Liu R, Sheng J, Liao J, Wang Y, Pan E, Guo W, Pu Y and Yin L: Differential expression profles of microRNAs as potential biomarkers for the early diagnosis of esophageal squamous cell carcinoma. Oncol Rep 29: 169-176, 2013.

5. Leite KR, Tomiyama A, Reis ST, Sousa-Canavez JM, Sañudo A, Camara-Lopes LH and Srougi M: MicroRNA expression profles in the progression of prostate cancer-from high-grade prostate intraepithelial neoplasia to metastasis. Urol Oncol 31: 796-801, 2013.

6. Li Y, Shao G, Zhang M, Zhu F, Zhao B, He C and Zhang Z: miR-124 represses the mesenchymal features and suppresses metastasis in Ewing sarcoma. Oncotarget 8: 10274-10286, 2017.

7. Schwentner R, Herrero-Martin D, Kauer MO, Mutz CN, Katschnig AM, Sienski G, Alonso J, Aryee DN and Kovar H: The role of miR-17-92 in the miRegulatory landscape of Ewing sarcoma. Oncotarget 8: 10980-10993, 2017.

8. Kawano M, Tanaka K, Itonaga I, Iwasaki T and Tsumura H: MicroRNA-301a promotes cell proliferation via PTEN targeting in Ewing's sarcoma cells. Int J Oncol 48: 1531-1540, 2016.

9. Esposito F, Tornincasa M, Pallante P, Federico A, Borbone E, Pierantoni GM and Fusco A: Down-regulation of the miR-25 and miR-30d contributes to the development of anaplastic thyroid carcinoma targeting the polycomb protein EZH2. J Clin Endocrinol Metab 97: E710-E718, 2012.

10. Yu H, Lin X, Wang F, Zhang B, Wang W, Shi H, Zou B and Zhao J: Proliferation inhibition and the underlying molecular mechanisms of microRNA-30d in renal carcinoma cells. Oncol Lett 7: 799-804, 2014.

11. Chen D, Guo W, Qiu Z, Wang Q, Li Y, Liang L, Liu L, Huang S, Zhao Y and He X: MicroRNA-30d-5p inhibits tumour cell proliferation and motility by directly targeting CCNE2 in non-small cell lung cancer. Cancer Lett 362: 208-217, 2015.

12. Kobayashi N, Uemura H, Nagahama K, Okudela K, Furuya M, Ino Y, Ito Y, Hirano H, Inayama Y, Aoki I, et al: Identification of $\mathrm{miR}-30 \mathrm{~d}$ as a novel prognostic maker of prostate cancer. Oncotarget 3: 1455-1471, 2012.

13. Li H, Zhang K, Liu LH, Ouyang Y, Bu J, Guo HB and Xiao T: A systematic review of matrix metalloproteinase 9 as a biomarker of survival in patients with osteosarcoma. Tumour Biol 35: 5487-5491, 2014.

14. Wang J, Shi Q, Yuan TX, Song QL, Zhang Y, Wei Q, Zhou L, Luo J, Zuo G, Tang M, et al: Matrix metalloproteinase 9 (MMP-9) in osteosarcoma: Review and meta-analysis. Clin Chim Acta 433: 225-231, 2014.
15. Shang HS, Chang JB, Lin JH, Lin JP, Hsu SC, Liu CM, Liu JY, Wu PP, Lu HF, Au MK and Chung JG: Deguelin inhibits the migration and invasion of U-2 OS human osteosarcoma cells via the inhibition of matrix metalloproteinase-2/-9 in vitro. Molecules 19: 16588-16608, 2014

16. Vinatier D, Dufour P and Subtil D: Caspases: Pharmacological manipulation of cell death. Eur J Obstet Gynecol Reprod Biol 67: 85-102, 1996.

17. Norbury CJ and Zhivotovsky B: DNA damage-induced apoptosis. Oncogene 23: 2797-2808, 2004.

18. Soldani C and Scovassi AI: Poly(ADP-ribose) polymerase-1 cleavage during apoptosis: An update. Apoptosis 7: 321-328, 2002.

19. Orrenius S: Mitochondrial regulation of apoptotic cell death. Toxicol Lett 149: 19-23, 2004.

20. Scorrano L and Korsmeyer SJ: Mechanisms of cytochrome c release by proapoptotic Bcl-2 family members. Biochem Biophys Res Commun 304: 437-444, 2003.

21. Meeran SM and Katiyar SK: Grape seed proanthocyanidins promote apoptosis in human epidermoid carcinoma A431 cells through alterations in Cdki-Cdk-cyclin cascade, and caspase-3 activation via loss of mitochondrial membrane potential. Exp Dermatol 16: 405-415, 2007.

22. Zenali MJ,Zhang PL, Bendel AE and Brown RE: Morphoproteomic confrmation of constitutively activated mTOR,ERK, and NF-kappaB pathways in Ewing family of tumors. Ann Clin Lab Sci 39: 160-166, 2009.

23. Roskoski R Jr: ERK1/2 MAP kinases: Structure, function and regulation. Pharmacol Res 66: 105-143, 2012.

24. Datta SR, Brunet A and Greenberg ME: Cellular survival: A play in three Akts. Genes Dev 13: 2905-2927, 1999.

25. Toretsky JA,Thakar M,Eskenazi AEandFrantzCN: Phosphoinositide 3-hydroxide kinase blockade enhances apoptosis in the Ewing's sarcoma family of tumors. Cancer Res 59: 5745-5750, 1999.

26. Balamuth NJ and Womer RB: Ewing's sarcoma. Lancet Oncol 11: 184-192, 2010.

27. Iwamoto Y: Diagnosis and treatment of Ewing's sarcoma. Jpn J Clin Oncol 37: 79-89, 2007.

28. Gorlick R, Janeway K, Lessnick S, Randall RL and Marina N; COG bone tumor committee: Children's oncology group's 2013 blueprint for research: Bone tumors. Pediatr Blood Cancer 60: 1009-1015, 2013.

29. Bartel DP: MicroRNAs: Genomics, biogenesis, mechanism, and function. Cell 116: 281-297, 2004.

30. Lee RC, Feinbaum RL and Ambros V: The C. elegans heterochronic gene lin-4 encodes small RNAs with antisense complementarity to lin-14. Cell 75: 843-854, 1993.

31. Šulc M, Marín RM, Robins HS and Vaníček J: PACCMIT/PACCMIT-CDS: Identifying microRNA targets in 3 UTRs and coding sequences. Nucleic Acids Res 43: W474-W479, 2015.

32. Fang Z, Tang J, Bai Y, Lin H, You H, Jin H, Lin L, You P, Li J, Dai Z, et al: Plasma levels of microRNA-24, microRNA-320a, and microRNA-423-5p are potential biomarkers for colorectal carcinoma. J Exp Clin Cancer Res 34: 86, 2015.

33. Nishikawa R, Goto Y, Kurozumi A, Matsushita R, Enokida H, Kojima S, Naya Y, Nakagawa M, Ichikawa T and Seki N: MicroRNA-205 inhibits cancer cell migration and invasion via modulation of centromere protein $\mathrm{F}$ regulating pathways in prostate cancer. Int J Urol 22: 867-877, 2015.

34. Volinia S, Calin GA, Liu CG, Ambs S, Cimmino A, Petrocca F, Visone R, Iorio M, Roldo C, Ferracin M, et al: A microRNA expression signature of human solid tumors defines cancer gene targets. Proc Natl Acad Sci USA 103: 2257-2261, 2006.

35. Zhang Y, Yang WQ, Zhu H, Qian YY, Zhou L, Ren YJ, Ren XC, Zhang L, Liu XP, Liu CG, et al: Regulation of autophagy by miR-30d impacts sensitivity of anaplastic thyroid carcinoma to cisplatin. Biochem Pharmacol 87: 562-570, 2014.

36. Gaziel-Sovran A, Segura MF, Di Micco R, Collins MK, Hanniford D, Vega-Saenz de Miera E, Rakus JF, Dankert JF, Shang S, Kerbel RS, et al: miR-30b/30d regulation of GalNAc transferases enhances invasion and immunosuppression during metastasis. Cancer Cell 20: 104-118, 2011.

37. Yao J, Liang L, Huang S, Ding J, Tan N, Zhao Y, Yan M, Ge C, Zhang Z, Chen T, et al: MicroRNA-30d promotes tumor invasion and metastasis by targeting Galphai2 in hepatocellular carcinoma. Hepatology 51: 846-856, 2010.

This work is licensed under a Creative Commons Attribution-NonCommercial-NoDerivatives 4.0 International (CC BY-NC-ND 4.0) License. 